$R M x A C, \mathbf{5 3}, 50-51$ (2021)

(C) 2021: Instituto de Astronomía, Universidad Nacional Autónoma de México

https://doi.org/10.22201/ia.14052059p.2021.53.13

\title{
AUTOMATIC ROTATIONAL SKY QUALITY METER (R-SQM) - ANALYSIS
}

\author{
Mohammad S. Niaei ${ }^{1,2}$, Cahit Yeşilyaprak ${ }^{1,2}$, Esragül Atalay ${ }^{1,2}$, and Emre Doğan ${ }^{1}$
}

RESUMEN

Se muestra la estadística a largo plazo del Observatorio de Anatolia del Este (DAG) detallando las condiciones de brillo del cielo de dicho emplazamiento, utilizando un sistema R-SQM. Tanto Python V3 como algunos otros módulos de Python se usaron para recoger y almacenar los datos en un servidor SQL siendo la visualización realizada por un servidor web utilizando HTML, PHP y Javascript.

\section{ABSTRACT}

Here we share the long term visualization and statistics of Easter Anatolian Observatory (DAG) Site's sky brightness conditions using an R-SQM system. Python V3 and some other Python modules were used to obtain and store the data in an SQL server and visualization done by a web server using HTML, PHP, and Javascript.

Key Words: atmospheric effects — instrumentation: miscellaneous — site testing

\section{INTRODUCTION}

The sky brightness of an observatory is an essential parameter for the site. Every professional observatory has a rough value for its sky brightness. An SQM is a device which can give the brightness of pointed direction within a solid angle(In this case 30 degrees).

R-SQM is composed by 4 SQM devices attached to a shaft which can rotate. SQMs are pointed to different altitudes (30, 45, 60 and 90) and can be rotated on its axis to achieve different azimuths. Using this device we obtained a good amount of data to be analyzed. Using all web technologies (Php, HTML, MySQL, and Javascript) we presented data.

\section{PERFORMANCE}

Automatic Rotational Sky Quality Meter (R$\mathrm{SQM})$ is a devices created for DAG project in Atatürk University Astrophysics Research and Application Center (ATASAM). R-SQM performance over approximately one year is satisfactory and data obtained can be used to present as the condition of the site. Please notice in winter regular maintenance or fast respond to problems was not possible.

\section{RESULTS}

Here we present R-SQM data obtained from DAG site. There are numerous types of data presentations such as polar and single plot, and other plots.

\footnotetext{
${ }^{1}$ Ataturk university, Astrophysics Research and Application Center. 02524 Erzurum/Turkey.

${ }^{2}$ Ataturk university, Department of Astronomy and Astrophysics. 02524 Erzurum/Turkey.
}

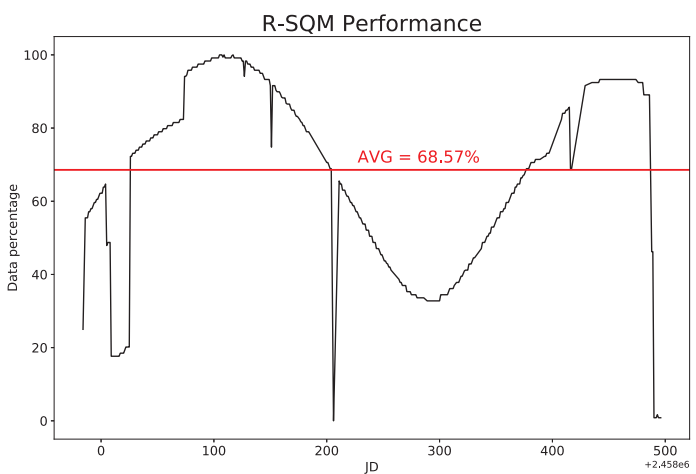

Fig. 1. R-SQM device performance.

\subsection{Sky Map}

The polar plot can present/map the whole sky's brightness. The plot can either display raw sqm magnitudes or calibrated/calculated magnitudes.

\subsection{Single Plot}

The single plot can present magnitude over time or angular distance from the north. In Fig. 3 the brightness is caused by the city of Erzurum (close to north). Before around 3780-degree (which means the device is facing azimuth 180 degrees after completing 10 cycles) the Moon's effect can be seen.

\subsection{Statitics}

These statistics were driven from DAG site at Alt-Az $(45,180)$ and can be interpreted with Fig. 4.

\section{CONCLUSION}

With this study we found yearly, monthly and seasonal average whole/partial sky brightnesses of DAG site. 


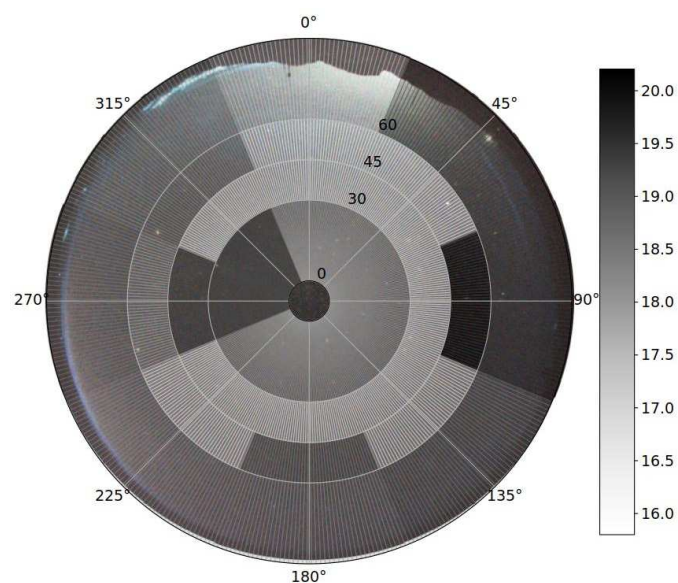

Fig. 2. DAG Sky map.

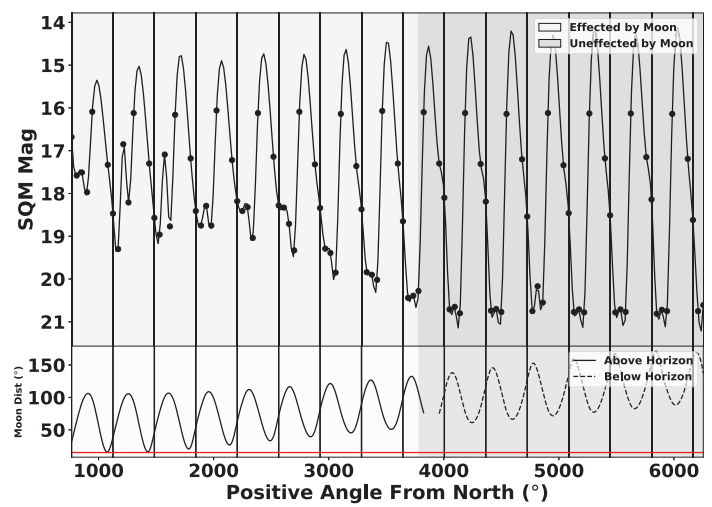

Fig. 3. Single plot of SQM-45. Up: Sky brightness. Black vertical lines indicate 315 degrees from the north which is the direction of the city of Erzurum. Down: Moon distance from SQM. The red line indicates the angular distance where the Moon is withing SQM's field of view.

TABLE 1

YEARLY AVERAGE

\begin{tabular}{ccccc} 
Year & Min & Avg & Max & Stdv \\
\hline 2017 & 15.01 & 19.24 & 22.97 & 1.72 \\
2018 & 15.01 & 18.88 & 22.93 & 1.44 \\
2019 & 15.37 & 20.22 & 22.13 & 1.13 \\
\hline
\end{tabular}

Using R-SQM we were able to map the sky brightness of DAG site and log-term results show DAG's sky is dark enough for its capacity.

As shown in Fig. 2 and Fig. 3 this device is capable to map the sky and using this data observation plans can be done more precisely.
TABLE 2

MONTHLY AVERAGE

\begin{tabular}{ccccc} 
Month & Min & Avg & Max & Stdv \\
\hline 1 & 15.10 & 18.28 & 22.12 & 1.25 \\
2 & 15.03 & 18.26 & 20.95 & 1.17 \\
3 & 15.43 & 17.97 & 20.03 & 1.27 \\
4 & 15.06 & 18.80 & 20.38 & 1.11 \\
5 & 15.09 & 18.58 & 20.55 & 1.14 \\
6 & 15.26 & 18.98 & 21.20 & 1.10 \\
7 & 15.01 & 19.53 & 21.40 & 1.51 \\
8 & 15.08 & 20.05 & 21.29 & 1.31 \\
9 & 15.01 & 19.52 & 21.29 & 1.50 \\
10 & 15.41 & 19.20 & 21.53 & 1.66 \\
11 & 15.01 & 19.44 & 22.97 & 1.63 \\
12 & 15.09 & 19.22 & 22.93 & 1.63 \\
\hline
\end{tabular}

TABLE 3

SEASONAL AVERAGE

\begin{tabular}{ccccc} 
Season & Min & Avg & Max & Stdv \\
\hline Winter & 15.03 & 18.23 & 22.12 & 1.23 \\
Spring & 15.06 & 18.77 & 21.20 & 1.13 \\
Summer & 15.01 & 19.73 & 21.40 & 1.46 \\
Autumn & 15.01 & 19.28 & 22.97 & 1.64 \\
\hline
\end{tabular}

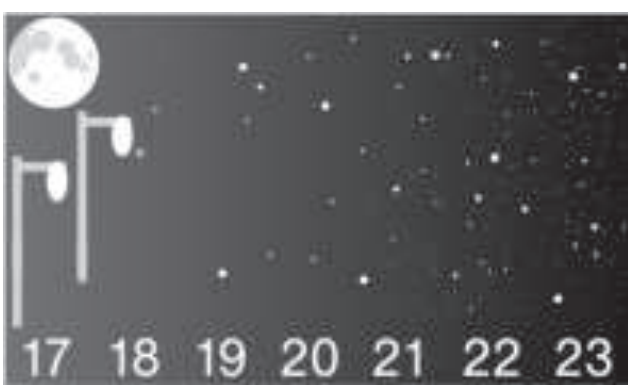

Fig. 4. SQM brighness rough meaning.

Acknowledgments: This study is supported by DAG Project (Project ID: 2011K120230), Atatürk Universtiy and ATASAM. Authors are grateful to DAG Project, Atatürk Universtiy and ATASAM for continuous and valuable supports.

We thank our colleagues from Atatürk University Astrophysics Research and Application Center (ATASAM) who assisted the research.

\section{REFERENCES}

Dogan, E., Ozbaldan, E. E., Shameoni, N. M., \& Yesilyaprak, C. 2016, RMxAC, 48, 31 SQM-LU Operator's Manual - Unihedron, (pp. 11, 14) 